\title{
Are there causal vacuum solutions with the symmetries of the Gödel universe in higher-derivative gravity?
}

\author{
A. J. Accioly \\ Instituto de Física, Universidade Federal do Rio de Janeiro, Caixa Postal 68528, Rio de Janeiro, CEP21944 Rio de Janeiro, Brazil \\ and Instituto de Física Teórica, Universidade Estadual Paulista, Rua Pamplona No. 145, São Paulo, CEP 01405, São Paulo, Brazil* \\ G. E. A. Matsas \\ Instituto de Física Teórica, Universidade Estadual Paulista, Rua Pamplona No. 145, Săo Paulo, CEP 01405, São Paulo, Brazil
}

(Received 6 May 1988)

\begin{abstract}
A globally causal vacuum solution with the symmetries of the Gödel universe is obtained in the framework of higher-derivative gravity. This very peculiar and rare result has no similar one in standard general relativity. The Rebouças-Tiomno universe, i.e., the unique completely causal Gödel-type solution of Einstein's field equations with an algebraic Segrè characteristic [(1,11)1], is shown to be a special case of our solution. We also show that our model is an embedding of the three-dimensional anti-de Sitter universe in four-dimensional space-time.
\end{abstract}

\section{INTRODUCTION}

In spite of the fact that all available evidence from experiments in macrophysics attests to the validity of Einstein's general theory of relativity as a description of gravitational interaction, it is highly desirable, for the sake of the unity and consistency of physics, that we can quantize gravity. Undoubtedly, some unification between (essentially) microphysics (quantum mechanics) and macrophysics (general relativity) must be part of nature's design.

At present, the $R+R^{2}$ theory of gravity has been suggested as a possible solution to the infinities plaguing the quantization of general relativity. The action for gravitation is given by

$I=\int d^{4} x \sqrt{-g}\left[\frac{R}{2 \kappa}-\frac{\Lambda}{\kappa}+\alpha R^{2}+\beta R_{\mu \nu} R^{\mu \nu}\right]$,

where $\alpha$ and $\beta$ are dimensionless coupling constants (in natural units) and $\kappa$ and $\Lambda$ are the Einstein and cosmological constants, respectively. The corresponding field equations are given by

$$
\begin{aligned}
\widetilde{G}_{\mu \nu}= & 0, \\
\widetilde{G}_{\mu \nu}= & (1 / \kappa)\left(R_{\mu \nu}-\frac{1}{2} R g_{\mu \nu}\right)+(\Lambda / \kappa) g_{\mu \nu} \\
& +\alpha\left(-R^{2} g_{\mu \nu}+4 R R_{\mu \nu}-4 g_{\mu v} \square R+4 \nabla_{\nu} \nabla_{\mu} R\right) \\
& +\beta\left(-2 \square R_{\mu \nu}-R_{\rho \theta} R^{\rho \theta} g_{\mu \nu}\right. \\
& \left.+4 R_{\mu \rho \theta v} R^{\rho \theta}-g_{\mu \nu} \square R+2 \nabla_{\nu} \nabla_{\mu} R\right)
\end{aligned}
$$

Not only is (1.1) renormalizable, ${ }^{1,2}$ but it is also asymptotically free. ${ }^{3,4}$ In spite of these promising attributes, (1.1) apparently suffers from the malaise of nonunitarity, because the bare propagator of the graviton field contains an undesirable ghost; i.e., a negative residue spin-2 pole.
Nevertheless, it is possible that this defect may be removed by means of radiation corrections. ${ }^{4,5}$ Consequently, the quantum interest concerning these quadratic Lagrangian theories is well suited.

On the other hand, from a classical viewpoint, higherderivative gravity may be considered as a possible generalization of general relativity. It seems natural then to investigate whether or not the aforementioned theory can predict some results not expected to be found in the standard general relativity.

As is well known, one of the most intriguing problems in general relativity emerges when we analyze the socalled Gödel-type universes, that is, models that are defined by the line element ${ }^{6}$

$$
d s^{2}=[d t+H(r) d \Phi]^{2}-D^{2}(r) d \Phi^{2}-d r^{2}-d z^{2},
$$

which in general admit closed timelike curves. In truth, it is difficult to resist the appealing idea of traveling into one's own past and thus conceivably influencing one's own history.

Recently, Rebouças and Tiomno ${ }^{7}$ have demonstrated that the necessary and sufficient conditions for a Gödeltype metric to be space-time homogeneous are

$$
\frac{H^{\prime}}{D}=\mathrm{const} \equiv 2 \Omega, \quad \frac{D^{\prime \prime}}{D}=\mathrm{const} \equiv m^{2} .
$$

Otherwise, it is not difficult to show, from their work, that vacuum solutions of the Gödel type related to space-time homogeneous models are not allowed in the context of general relativity. The following interesting questions can now be posed: are there vacuum solutions concerning the homogeneous Gödel-type models in the higher-derivative gravity framework? In case of an affirmative answer, what about the existence of causal anomalies (in the form of closed timelike curves) in these solutions? 
Our aim here is to answer the above questions. We begin by exhibiting a void solution with the symmetries of the Gödel universe in Sec. II. As such, it is rather remarkable, having no analogs in the context of general relativity. In Sec. III we show that our solution is globally causal, although it is not stably causal. We conclude the paper with some interesting observations about the results obtained.

\section{A VACUUM SOLUTION WITH THE SYMMETRIES OF THE GÖDEL UNIVERSE VIA HIGHER-DERIVATIVE GRAVITY}

Inserting the Gödel-type metric (1.4) in the higherderivative gravity field equations (1.2), the following set of equations is obtained:

$$
\begin{aligned}
& \widetilde{G}_{00}=\frac{1}{\kappa}\left[-\frac{1}{2}\left[\frac{H^{\prime}}{D}\right]^{2}-\frac{R}{2}\right]+\frac{\Lambda}{\kappa}+\alpha\left[-R^{2}-2 R\left(H^{\prime} / D\right)^{2}+\left(4 D^{\prime} R^{\prime}\right) / D+4 R^{\prime \prime}\right] \\
& +\beta\left\{-\frac{15}{4}\left[\frac{H^{\prime}}{D}\right]^{4}-3 \frac{H^{\prime}}{D}\left[\frac{H^{\prime}}{D}\right]^{\prime \prime}-\frac{3}{2}\left[\left(\frac{H^{\prime}}{D}\right)^{\prime}\right]^{2}-3 \frac{D^{\prime}}{D} \frac{H^{\prime}}{D}\left(\frac{H^{\prime}}{D}\right)^{\prime}+6 \frac{D^{\prime \prime}}{D}\left(\frac{H^{\prime}}{D}\right)^{2}\right. \\
& \left.-2\left(\frac{D^{\prime \prime}}{D}\right)^{2}-2\left[\frac{D^{\prime \prime}}{D}\right]^{\prime \prime}-2 \frac{D^{\prime}}{D}\left(\frac{D^{\prime \prime}}{D}\right)^{\prime}\right\}=0 \\
& \widetilde{G}_{11}=\frac{1}{\kappa}\left[-\frac{1}{2}\left[\frac{H^{\prime}}{D}\right]^{2}+\frac{D^{\prime \prime}}{D}+\frac{R}{2}\right]-\frac{\Lambda}{\kappa}+\alpha\left[-R^{2}-R\left(H^{\prime} / D\right)^{2}-\left(4 D^{\prime} R^{\prime}\right) / D\right] \\
& +\beta\left\{-\frac{9}{4}\left(\frac{H^{\prime}}{D}\right)^{4}-5 \frac{D^{\prime}}{D} \frac{H^{\prime}}{D}\left[\frac{H^{\prime}}{D}\right)^{\prime}+4 \frac{D^{\prime}}{D}\left[\frac{D^{\prime \prime}}{D}\right)^{\prime}+\frac{1}{2}\left[\left(\frac{H^{\prime}}{D}\right)^{\prime}\right]^{2}-\frac{H^{\prime}}{D}\left(\frac{H^{\prime}}{D}\right)^{\prime \prime}\right. \\
& \left.+4 \frac{D^{\prime \prime}}{D}\left(\frac{H^{\prime}}{D}\right)^{2}-2\left(\frac{D^{\prime \prime}}{D}\right)^{2}\right\}=0 \text {, } \\
& \widetilde{G}_{22}=\frac{1}{\kappa}\left[-\frac{1}{2}\left(\frac{H^{\prime}}{D}\right)^{2}+\frac{D^{\prime \prime}}{D}+\frac{R}{2}\right]-\frac{\Lambda}{\kappa}+\alpha\left[-R^{2}-4 R^{\prime \prime}-R\left(H^{\prime} / D\right)^{2}\right] \\
& +\beta\left\{-\frac{9}{4}\left[\frac{H^{\prime}}{D}\right]^{4}-\frac{9}{2}\left[\left(\frac{H^{\prime}}{D}\right]^{\prime}\right]^{2}+4\left(\frac{D^{\prime \prime}}{D}\right)^{\prime \prime}-2\left(\frac{D^{\prime \prime}}{D}\right)^{2}-\frac{H^{\prime}}{D} \frac{D^{\prime}}{D}\left[\frac{H^{\prime}}{D}\right)^{\prime}\right. \\
& \left.-5 \frac{H^{\prime}}{D}\left(\frac{H^{\prime}}{D}\right)^{\prime \prime}+4\left(\frac{H^{\prime}}{D}\right)^{2} \frac{D^{\prime \prime}}{D}\right\}=0 \text {, } \\
& \widetilde{G}_{33}=\frac{1}{\kappa} \frac{R}{2}-\frac{\Lambda}{\kappa}+\alpha\left[R^{2}-4 R^{\prime \prime}-\left(4 D^{\prime} R^{\prime}\right) / D\right] \\
& +\beta\left\{\frac{3}{4}\left[\frac{H^{\prime}}{D}\right]^{4}-\frac{3}{2}\left[\left(\frac{H^{\prime}}{D}\right]^{\prime}\right]^{2}+2\left(\frac{D^{\prime \prime}}{D}\right)^{2}-2 \frac{D^{\prime \prime}}{D}\left[\frac{H^{\prime}}{D}\right)^{2}-\frac{H^{\prime}}{D}\left(\frac{H^{\prime}}{D}\right)^{\prime \prime}+2\left(\frac{D^{\prime \prime}}{D}\right)^{\prime \prime}\right. \\
& \left.-\frac{D^{\prime}}{D} \frac{H^{\prime}}{D}\left[\frac{H^{\prime}}{D}\right]^{\prime}+2 \frac{D^{\prime}}{D}\left[\frac{D^{\prime \prime}}{D}\right]^{\prime}\right\}=0 \\
& \widetilde{G}_{02}=-\frac{1}{2 \kappa}\left[\frac{H^{\prime}}{D}\right]^{\prime}+\alpha\left[-2 R\left(H^{\prime} / D\right)^{\prime}-\left(2 H^{\prime} R^{\prime}\right) / D\right] \\
& +\beta\left[-\left(\frac{H^{\prime}}{D}\right]^{\prime \prime \prime}-9\left(\frac{H^{\prime}}{D}\right)^{2}\left(\frac{H^{\prime}}{D}\right)^{\prime}+3\left(\frac{H^{\prime}}{D}\right)^{\prime} \frac{D^{\prime \prime}}{D}+4 \frac{H^{\prime}}{D}\left(\frac{D^{\prime \prime}}{D}\right)^{\prime}+\left(\frac{H^{\prime}}{D}\right)^{\prime}\left(\frac{D^{\prime}}{D}\right)^{2}-\frac{D^{\prime}}{D}\left[\frac{H^{\prime}}{D}\right]^{\prime \prime}\right]=0,
\end{aligned}
$$


where

$$
R=\frac{1}{2}\left(H^{\prime} / D\right)^{2}-\left(2 D^{\prime \prime}\right) / D
$$

and the primes denote differentiation with respect to $r$.

The solution of these equations, in the case of homogeneous Gödel-type universes (1.5), can be presented in the form

$$
\begin{aligned}
d s^{2}= & d t^{2}+\frac{2}{\Omega} \sinh ^{2}(\Omega r) d \Phi d t-d r^{2}-d z^{2} \\
& -\frac{1}{\Omega^{2}} \sinh ^{2}(\Omega r) d \Phi^{2}
\end{aligned}
$$

with

$$
\Omega^{2}=\frac{m^{2}}{4}=\frac{1}{8(3 \alpha+\beta)_{\kappa}}=-\frac{2}{3} \Lambda .
$$

We have thus succeeded in finding a vacuum solution (counting the cosmological constant as "vacuum") of the Gödel type in the context of the fourth-order gravity theory that is not a solution of the corresponding Einstein's equations. As such it is rather unusual; it is also interesting because it relates Newton's constant, $\alpha, \beta$, and the value of the cosmological constant. In a sense it establishes a link between the microphysics and the macrophysics.

By a trivial change of variables $\left[1 / 8(3 \alpha+\beta) \kappa \rightarrow \kappa e^{2} / 2\right.$, $\frac{2}{3} \Lambda \rightarrow \Lambda / 2$ ], the homogeneous Rebouças-Tiomno universe, ${ }^{7-13}$ i.e., the unique completely causal Gödel-type solution of Einstein's field equations with an algebraic Segrè characteristic $[(1,11) 1]$, can be recovered. Therefore this universe is nothing but a rather special case of our solution.

\section{A NOTE ABOUT THE NONEXISTENCE OF CLOSED TIMELIKE CURVES CONCERNING THE HOMOGENEOUS GÖDEL-TYPE UNIVERSES IN THE LIMITING CASE $m^{2}=4 \Omega^{2}$}

We are ready now to focus our attention on the problem of the existence of causal anomalies (in the form of closed timelike curves) in our solution. The presence of closed timelike curves of the Gödel type, that is, the circles defined by $t, z, r=$ const, depends on the behavior of the function

$$
f(r)=D^{2}(r)-H^{2}(r) .
$$

Indeed, if $f(\boldsymbol{r})$ becomes negative for a certain range of values of $r\left(r_{1}<r<r_{2}\right.$, say), Gödel's circles are closed timelike curves. In the specific situation we are analyzing, $f(r)$ is given by

$$
f(r)=\frac{1}{\Omega^{2}} \sinh ^{2}(\Omega r) .
$$

Thus, we can guarantee that there is no breakdown of causality of the Gödel type (circles) in our model. We cannot assure, of course, that all possible curves are causal by looking over the causality of $r, t, z,=$ const curves. In order to face this situation we introduce new coordinates $\phi$ and $R$ defined by

$$
\phi=\Phi-\Omega t, \quad \Omega R=\sinh (\Omega r),
$$

and rewrite the metric for the hyperbolic family of space-times (2.3) in the form

$$
\begin{aligned}
d s^{2}= & \left(1+\Omega^{2} R^{2}\right) d t^{2}-\left(1+\Omega^{2} R^{2}\right)^{-1} d R^{2} \\
& -R^{2} d \phi^{2}-d z^{2} .
\end{aligned}
$$

This metric is nothing but an ordinary embedding of the three-dimensional anti-de Sitter metric in the fourdimensional spacetime. As a result, this space does not contain any closed timelike lines. ${ }^{14}$ It has the topology of $R^{4}$.

We shall now prove that the $m^{2}=4 \Omega^{2}$ space-time is not stably causal. In order to accomplish this, we first introduce new coordinates $t^{\prime}, x$, and $y$ defined by ${ }^{7}$

$$
\begin{aligned}
& \tan \left[\frac{\Phi}{2}+\left(t^{\prime}-t\right)\right]=e^{-m r} \tan (\Phi / 2), \\
& e^{m x}=\cosh (m r)+\sinh (m r) \cos \Phi, \\
& m y e^{m x}=\sinh (m r) \sin \Phi,
\end{aligned}
$$

and rewrite the line element (2.3) in the form

$$
d s^{2}=\left(d t^{\prime}+e^{m x} d y\right)^{2}-e^{2 m x} d y^{2}-d x^{2}-d z^{2},
$$

where $-\infty<t^{\prime}, x, y, z<+\infty$, rendering explicit that the manifold has been endowed with the $R^{4}$ topology. On the other hand, as is well known, a space-time is stably causal if and only if it admits a global time function. ${ }^{15}$ From Eq. (3.6) we get, for the function $F=t^{\prime}$,

$$
F^{, \mu} F_{, \mu}=0 \text {. }
$$

Thus, the gradient $F_{, \mu}$ is null-like provided $m^{2}=4 \Omega^{2}$. It follows then, that $F$ is not a global time function. As a consequence the space-time is not stably causal. In a sense we can say that it is "on the verge" of displaying the breakdown of causality.

\section{FINAL REMARKS}

We have shown, through a specific example, the potentialities of higher-derivative gravity when evaluated from a classical point of view. In particular, this theory has bequeathed to us a very peculiar and rare result: a completely causal vacuum solution with the symmetries of the Gödel universe.

Could it be that the solution we have found is just flat space or some other simple space? One can demonstrate that this space-time has a seven-parameter maximal group of motion $\left(G_{7}\right)$ while the remaining homogeneous Gödel-type metrics have a $G_{5}$ (Ref. 8). Otherwise, it is a well-established fact that solutions with a $G_{7}$ of motions are rather uncommon. ${ }^{16}$

\section{ACKNOWLEDGMENTS}

A.J.A. gratefully acknowledges financial support from the Conselho Nacional de Desenvolvimento Científico e Tecnológico (CNPq). G.E.A.M. was supported by Fundação de Amparo e Pesquisa do Estado de São Paulo (FAPESP), a Brazilian agency. A.J.A. was partially supported by FAPESP. 
*Permanent address and address for correspondence.

${ }^{1}$ K. S. Stelle, Phys. Rev. D 16, 953 (1977).

${ }^{2}$ B. L. Voronov and I. V. Tyutin, Yad. Fiz. 39, 998 (1984) [Sov. J. Nucl. Phys. 39, 631 (1984)].

${ }^{3}$ E. S. Fradkin and A. A. Tseytlin, Nucl. Phys. B201, 169 (1982).

4J. Antoniadis and E. Tomboulis, Phys. Rev. D 33, 2756 (1986).

${ }^{5}$ A. Salam and J. Strathdee, Phys. Rev. D 18, 4480 (1978).

${ }^{6}$ M. J. Rebouças and J. Tiomno, Nuovo Cimento B 90, 204 (1985).

${ }^{7}$ M. J. Rebouças and J. Tiomno, Phys. Rev. D 28, 1251 (1983).

${ }^{8}$ A. F. F. Teixeira, M. J. Rebouças, and J. E. Åman, Phys. Rev. D 32, 3309 (1985).

${ }^{9}$ M. J. Reboucas, J. E. Åman, and A. F. F. Teixeira, J. Math. Phys. 27, 1370 (1986).
${ }^{10}$ M. J. Rebouças and A. F. F. Teixeira, Phys. Rev. D 34, 2985 (1986).

${ }^{11}$ J. D. Oliveira, A. F. F. Teixeira, and J. Tiomno, Phys. Rev. D 34, 3661 (1986).

${ }^{12}$ M. J. Rebouças and J. E. Åman, J. Math. Phys. 28, 888 (1987).

${ }^{13}$ M. O. Calvão, M. J. Rebouças, A. F. F. Teixeira, and W. M. Silva, Jr., J. Math. Phys. (to be published).

${ }^{14} \mathrm{~S}$. W. Hawking and G. F. R. Ellis, The Large Scale Structure of Space-Time (Cambridge University Press, Cambridge, England, 1973).

${ }^{15}$ S. W. Hawking, Proc. R. Soc. London A308, 433 (1969).

${ }^{16} \mathrm{See}$, for instance, D. Kramer, H. Stephani, M. MacCallum, and E. Herlt, Exact Solutions of Einstein's Field Equations (Cambridge University Press, Cambridge, England, 1980). 